Hæmorrhage by contre coup was diagnosticated, and the clot successfully removed at my suggestion by Dr. Homans, perfect recovery following. The hæmorrhage was subdural and involved the motor tract and temporal region, most copious over the facial area.

In the present case we had to do with a hæmorrhage in such location, and so quickly poured out, that by the time the symptoms were sufficiently marked to point with any definiteness to the seat of the lesion, the base of the skull had been flooded, and the vertebral canal so far invaded as to cause severe pressure upon the medulla oblongata. The only excuse for operation here would seem to be the otherwise hopeless condition of the patient. Upon this point the personal equation varies greatly, but the prevailing feeling among the consultants in this case was that while any hope of relief, however faint, would justify the attempt in so desperate a situation, there was really absolutely no prospect of relief by surgical interference. The patient was on the verge of death, which the shock of operation might only precipitate, and remove the remote (certainly extremely remote) possibility of absorption. Ligature of the carotid artery was hardly advisable. This procedure, aimed to control, for example, middle meningeal hæmorrhage, though proposed by Forneaux, Roser, Simons and others, ${ }^{8}$ promises very little in these cases, as shown by Gallez ${ }^{4}$ in his recent contribution to the study of trephining, who points out that the hæmorrhage is not always controlled in this way, and that even if it be controljed, the consequences of the clot are not averted.

The question of trephining for hæmorrhage at the base, with or without fracture in this location, is one upon which study is not exhausted. Possibly future experience may lead to less conservatism in this class of cases, but we do not gain sufficient encouragement from the present status of cerebral surgery to afford hope that drainage of the base of the skull, difficult at the best (particularly on account of pressure down. wards of the cranial contents) can be satisfactorily established in so desperate a case as this, in which the vertebral canal has been already invaded.

Gallez, ${ }^{6}$ in commenting upon this point, after speaking of the comparative hopelessness of operations for very extensive hæmorrhage, uses the following language: "It is even less justifiable to undertake operation when symptoms exist of compression of the bulb, and of the nerves at the base of the brain; dilatation and paresis of the pupils, or rapid pulse (caused by paralysis of the pueumogastric). The extravasation is too diffuse."

Jacobson and Agneau are quoted by this writer in substantiating this view, the former authority adding that the condition points to extensive fracture. This would certainly in a majority of cases be true, and was to have been expected in our case, but whether fracture were present or not, the contra-indications to operation for hæmorrhage of this extent and seat, would seem absolute.

The autopsy, in the opinion of all present, certainly justified the conclusions drawn, both regarding the localization and the question of operation, the vital points in the case. The existence or non-existence of fracture had comparatively little practical bearing, either on the diagnosis, prognosis or treatment.

3 Lancet, 1885 ; Medical Times, October 17, 1883, page 548.
4 La Trepanation du Crâne, Paris, 1893.

5 Loc. cit., page 272 .

\section{MALIGNANT DISEASE OF THE RECTUM. ${ }^{1}$}

\author{
BY GEORGE W. GAY, M.D., \\ Surgeon to the Boston City Hospital.
}

THE brief paper which I venture to present to you to-night is a sort of running commentary upon some of the salient points of this disease and upon some of the cases met with in my practice. As there is no known permanent, radical cure for these affections, our efforts are necessarily directed to the management of the cases with a view to prolonging life, and to making it as endurable as possible while it lasts.

The first point to receive consideration in all diseases of the rectum is the diagnosis. I should not feel called upon to advise and to urge upon the younger members of this Society the very great importance of a digital and visual examination, had I not seen and made some very embarrassing mistakes by neglecting this important essential. Every hospital or consulting surgeon has met with cases of malignant disease of the anus and rectum which have been treated as piles, simply because no proper examination had ever been made.

A noted musician, whom the older portion of this community remembers with pleasure, came into my office some years ago; saying that he had the piles, and had been treated for them off and on for two or three years. He wanted to know what was the matter with him. I was shocked at his appearance; emaciated, sallow, weak, a wreck of his former robust self. A digital examination revealed a large mass of malignant disease situated just above the internal sphincter, which any one could easily have found, and the character of which could not have been mistaken for anything else. It need not be said that I received much undeserved credit in the case by simply telling him his real condition.

As further showing the importance of making a careful examination in cases of suspected disease of the rectum, I may allude to the case of a woman who was brought to me by a reputable physician for an opinion as to the character of a rectal tumor which he had detected by a vaginal examination alone. Epsom salts and warm-water injections removed the lump in a few days.

Both of these mistakes could have been easily avoided by simply making a digital examination of the rectum. The diagnosis should never be made upon subjective symptoms alone. The external parts should be carefully inspected, and the finger should be carried as far up the rectum as possible. A lady was put under my care several years ago by Dr. Brainard, for the removal of a small patch of malignant disease situated nearly or quite five inches from the anus. It was reached with difficulty by the finger, and a careful, thorough search was necessary for its detection. Hæmorrhage was the principal symptom.

A woman about fifty-two years of age is under my care at the preseut time, who for several weeks had pelvic pain and colic, which were greatly aggravated by laxatives or cathartics. Constipation was obstinate. Repeated vaginal and rectal examinations revealed only a uterine fibroid on the posterior surface of the womb, which, it was thought, might be the cause of the pain and constipation through pressure. Etherization relaxed the parts, so that this explanation was at once

1 Read before the Boston Society for Medical Observation, January 1,1894 . 
abandoned, and a fibrous stricture was detected, as far up the rectum as the fingers could be carried. It was annular, movable, firm, not hard nor nodular. Its character may be inferred from the fact, that the symptoms were promptly ameliorated by inunctions of oleate of mercury, and the administration of iodide of potash. These cases are sufficient to show the importance of a thorough digital examination.

The last case leads me to speak of the great value of an anæsthetic in all rectal affections located above the sphincters. Except in very thin people with lax tissues, it is not possible to make a thorough and satisfactory examination of the pelvic organs without an anæsthetic. This rule is emphatically true, as regards affections of the rectum. With the patient under ether in the lithotomy position, the sphincters are to be thoroughly stretched, and the rectum cleared by an enema if necessary. A digital examination can now be made to the best advantage to detect strictures, growths or ulcers. With a Sims's speculum and a horizontal light, a good view can be obtained of from four to six inches of the lower part of the rectum, which is the location of nine-tenths of its lesions.

Bougies, sounds and catheters are of little use in making a diagnosis of stricture of the rectum. They impinge upon the promontory of the sacrum, or get caught in a fold of the mucous membrane, thereby giving untrustworthy evidence as to the true condition of the part. The finger is the only reliable sound, and the diagnosis of those rather rare strictures located above its reach is to be made from the symptoms, such as colic, abdominal distention, etc.

The majority of abnormal growths found in the rectum of adults are either specific or malignant in character. Aside from these causes, strictures may be due to tuberculous or other inflammatory changes, or they may be traumatic in character. Neoplasms or strictures due to other causes than cancer or venereal disease, are not very common in our metropolitan hospitals. Dr. Kelsey, in the late edition of his admirable work upon diseases of the rectum, makes the statement that almost as many strictures of the rectum, not malignant, are non-venereal as are venereal. Considering the facts, that little dependence can generally be placed upon the therapeutic test of the nature of the affection; that the clinical appearances are not always sufficiently characteristic; and that histories of cases through ignorance, carelessness, or wilful deception, are proverbially unreliable, I am unable to understand this statement. Specific and venereal diseases permeate society to such an extent, and are met with in such unexpected places, that the older practitioners, who have seen a good deal of these affections, will hesitate to subscribe to the above statement.

I venture the assertion that strictures of the rectum in adults, non-malignant and non-venereal are not of ten met with in our large hospitals. It is by no means easy in every instance to decide at once as to which of the two most common classes the patient belongs, but the cases are rare which cannot fairly be ascribed to malignant or venereal causes.

A differential diagnosis is of much importance, as affecting the prognosis and treatment. Specific and other non-malignant strictures may, and not infrequently do, send their owners to the poorhouse; but it is only after many years of suffering that they find rest in the grave. On the other hand, malignant disease of the rectum usually runs its course in two to three years, and terminates fatally in the vast majority of cases. The exceptions to this rule are so rare, that $I$ have ascribed the recovery in two instances to a mistake in the diagnosis.

When a digital examination reveals a hard, nodular mass in the rectum, more or less immovable, involving the mucous, submucous and adjacent structures, and encroaching upon the lumen of the bowel, there can be little doubt as to the malignant character of the disease. It is safe to entertain the same opinion in cases of hard, ragged ulcerations with indurated edges, and deep attachments. Not infrequently a rather soft, friable upgrowth is met with, which bleeds easily, grows rapidly, and is liable to recurrence.

Malignant disease at the anus resembles epithelial growths in other regions of the body, presenting an ulcerated surface with or without an upgrowth, and having a hard indurated base. 'The surrounding skin usually presents a healthy appearance, rather than a sodden, fissured, or nodular surface, as is so frequently seen in cases of specific disease in this region.

A broad, general distinction between malignant and specific disease of the lower bowel is, that the latter involves the anus, while the former is located higher up, so that oftentimes an area of healthy mucous membrane separates the disease from the sphincters.

While the therapeutic test is usually disappointing and unreliable, yet occasionally it is very satisfactory. Four years ago I saw, with Dr. Joseph Stedman, a woman under forty years of age who had extensive disease of the rectum above the sphincters, which was hard, indurated, iuvolving adjacent tissus, thereby leading me to express the opinion, that it was maliguant in character. She was put upon the compound tincture of iodine, and given an unfavorable prognosis. She took the medicine faithfully for many months, and still continues its use at intervals. She is very well, and has evidently derived great benefit from the drug. In this case the diagnosis was wrong, but the treatment was right.

It goes without saying that the prognosis in malignant disease of the rectum, as in other parts of the body, is unfavorable. While there is no known cure for these affections, yet much can be done to prolong life in many cases, and nearly always to make it more endurable.

The treatment is palliative or radical. I think that the opinion is gaining ground in this country that the former is preferable in very many patients. The operation, or mode of treatment, which cures cancer, Las not been discovered. Hence the object of our efforts in the mauagement of the affections under consideration is to give the sufferers all the comfort possible. It does not always seem desirable to prolong life, and yet that is very properly one of the objects of the physician's work.

The treatment of this disease may be considered under three heads, namely : the alterative, the opium, and the operative methods. Whether one or all of these modes of treatment shall be pursued in any given case, depends upon circumstances.

A good illustration of the alterative method was seen in the case of a policeman about forty years of age, who had a malignant ulcerating mass three inches in diameter upon the anterior wall of the rectum above the sphincters. Its location precluded any radical operation, and the symptoms did not call for colotomy. He took from nine to twenty-seven drops of the com. 
pound tincture of iodine daily for upwards of two years; once or twice a day he took an enema containing from one to two grains of cocaine. He was thus enabled to do his work as patrolman for the above period. The time finally came when his strength began to fail ; and on the day that he was discharged from the force, he went into an adjoining room, and shot himself dead. He fully understood the hopeless nature of his disease; and the moment that he was disabled he took the management of the case into his own hands.

In view of the fact that this man was always better on resuming the treatment, his physicians could not but think, that it had a decided beneficial effect.

It is nearly three years since $I$ removed a growth two inches in diameter from the rectum of a man fortyseven years of age, by Cripps's operation. It was pronounced malignant by competent authority. The symptoms were pain and hæmorrhage. He has taken the iodine daily since the operation, and there has been no return of either symptoms or growth. I dread, however, to see this man enter my office, as I do any other person, upon whom I have operated for caucer; for it is probably only a question of time when a recurrence will take place.

Six years ago I removed a similar growth from the rectum of a middle-aged lady, by Cripps's operation. For tour years she took Chian turpentine, and drank Ypsilanti water constantly. There has been no recurrence of the hæmorrbage, or of the disease. I am inclined to ascribe the cure in this case to a mistake in the diagnosis, rather than to the treatment.

Many years ago Dr. Henry M. Field, of Newton, suggested to me the iodine method of treating malignant disease of the rectum. He thought that he had seen beneticial effects from its use; and so far as regards two cases, which I saw with him, I can confirm his opinion. For a long time I have given the drug to nearly all of my patients with malignant disease, wherever located, provided they could take it without disturbing the stomach. In many cases I could not see that any benefit was derived from its use. The rapid growing and markedly malignant ones, as might be supposed, derive less aid from it than others.

The natural course of malignant disease varies so much in different individuals, that it is extremely difficult to arrive at reliable conclusions as to the true value of any drug in checking or modifying the new growths. Chian turpentine, cundurango and many other articles have had their day, and have been discarded because they did not stand the test of experience. I do not claim anything for iodine. I use it, because I think it is our duty to give these unfortunate people the benefit of every possible aid; and for this particular purpose, I know of nothing better at present than the compound tincture of iodine.

Sooner or later most patients afflicted with malignant disease of the rectum are compelled to resort to opium to relieve their sufferings. 'The pain is usually due to obstructed peristalsis, colic as we call it, or to the pressure or other influence of the growth itself upon the nerves. The first is paroxysmal, and is coincident with the vermicular action of the bowels. The latter is a steady, grinding, boring, or aching pain, very wearing to the nervous system, and very distressing to bear. Both can be controlled to a certain extent with opium. Both are relieved temporarily by excision of the growth. Colotomy usually relieves the colic permanently, but may do the pressure-pain little, if any good.
A fair example of the opium treatment was recently under my care through the courtesy of Dr. Whittier. A man, sixty-eight years old, had suffered about two years with a cancer of the rectum. It was located well above the sphincters, hard, nodular, and involving the neighboring parts to a wide extent. Colic and pressurepains were severe. The liver was greatly enlarged by a secondary growth extending nearly to the umbilicus. Several surgeons were consulted at different times, and all very properly advised colotomy. The patient finally became so weak and exhausted from the progress of the disease, aggravated by the great difficulty experienced every week in his efforts to empty the bowels, that he realized the fact that something must be done. The opium and operative treatments were fully explained to him and he decided to try the former. At first a quarter of a grain of morphine given twice daily under the skin was sufficient to relieve most of the pain. This quantity was gradually increased until he took between three and four grains daily, with the effect of keeping him fairly comfortable. The bowels did not move for nine weeks after he began the morphine. His diet consisted of gruels, soups, and beef extracts. No clear or unmodified milk was given during this period. After some weeks of this complete obstruction, the fæces could be easily felt in the left iliac region. The abdomen never became distended to any extent, although very little flatus escaped for several weeks. The paresis of the intestines produced by the morphia was surprising. There was no vomiting, hiccoughs, delirium, nor headache. Thirst was urgent, and the mouth and throat were very dry and parched, in spite of all our efforts to prevent it. The emaciation became extreme. The bowels finally began to move of themselves, without aid of any sort; and during the last weeks of his life he had from two to six stools daily, thereby completely removing the collec. tion above the stricture. There was no hæmorrhage. $\mathrm{He}$ died from exhaustion. A seasonable colotomy would doubtless have enabled this man to keep about longer, and to have postponed the opium stage for a time. Whether his life would have been prolonged thereby, is a question not so easily decided.

Under the head of operative treatment there are three measures, which require consideration, namely, Cripps's and Kraske's operations and their modifications, and colotomy. The first is particularly adapted for cases in which the disease is limited to the first five inches of the bowel. It consists briefly in dividing both sphincters and rectum as high as the tip of the $\operatorname{cocc} x \mathrm{x}$, and then dissecting out the growth or diseased tissues.

Judging from a limited experience, this is a very satisfactory operation. The dangers are shock and hæmorrhage. I have never seen incontinence of gas or fæces to a troublesome degree follow this operation. It is especially indicated for the removal of growths which are limited in extent, movable, and not located over the urethra and prostate.

Kraske's operation is designed for the removal of growths located higher up than five inches, or above the reach of the finger. The sphincters are not divided. The bowel is reached through an incision over the sacrum and coccyx. The latter bone and a portion of the former is removed. The gut is divided above and below the diseased portion; the latter is removed; and the ends of the healthy bowel are joined with sutures or fastened in the wound, as the condition requires. 
The peritoneal cavity is usually opened in this operation, thereby adding auother element of danger from extravasation of fæces.

Finally, we come to the most satisfactory operation for the relief of the majority of cases, colotomy. This is now most frequently done in the left inguinal region, for the reason that the operation is more quickly and easily performed, the wound can be attended to far better by the patient, and the danger is no greater than in the loin. The relief to the obstructive pain or colic is usually complete and permanent. The pressure-pain may still require opiates or other treatment. Many lives are prolonged; and, more than that, they are made comparatively comfortable by this operation. In most cases the patient can keep himself clean and free from odor. A woman upon whom Dr. Bradford performed right lumbar colotomy worked for many months alongside another woman, without the latter ever suspecting that anything was the matter with her companion.

Kelsey's description of the operation is the best one I have seen. The incision parallel with Poupart's ligament is made about an inch from the left anterior superior spine. The peritoneal cavity is opened, and the colon brought out of the wound. The "bar" is made by joining the edges of the incision with a silver wire passed underneath the bowel. The intestine is then joined to the edges of the wound with sutures, and two or three days allowed to elapse before the bowel is opened. The operation is neither difficult nor dangerous, and in very many cases it is a most satisfactory one to both patient and surgeon.

By way of recapitulation it may be said, that the treatment of malignant disease of the rectum is essentially palliative. Colotomy is indicated to relieve obstructive colic, and it should be done early in order to save the patient's strength. The radical operations are indicated in the early stage, when the growth is limited in extent, free from deep adhesions and infiltration, and not infringing upon the urethra or prostate. Opium, the king of drugs, is to be avoided as long as possible, and given judiciously, in order that its beneficial effects may not be lost by over-doses before the time comes when it is needed the most. While the disease is incurable, very much can be done by judicious management to make the patient's life endurable and his death peaceful and easy.

\section{A CASE OF PULMONARY STENOSIS IN AN ADUL'T.}

BY OLIVER H. EVERETT, M.D., WORCESTER, MASS.

The following case is interesting in that the diagnosis was made incidentally, and not because the patient complained of any symptoms pointing to her heart. Furthermore, the rarity of the lesion in an adult makes it worthy of record, as well as the fact that the patient has attained her present age with so few symptoms. Professor Loomis, in reporting a similar case, ${ }^{1}$ speaks of it as one of the "curiosities of medicine."

Mrs. X., twenty-seven years old, a native of the State of New York, of Freuch-Canadian parentage, applied for admission to the Memorial Hospital, November 10, 1893, thinking she had typhoid fever, as

1 International Clinics, 1893, second series, vol. iv, p. 13. she had been taking care of a patient with that disease. She had a slightly elevated temperature, and was admitted for observation. She says she has always been fairly well, though never strong and cannot do bard work without getting tired and out of breath. Even as a girl she could not join in active plays. There is no history of rheumatism, chorea or other disease except scarlet fever and la grippe. When she was ten or twelve years old her dyspnœa became more marked; and in the course of the next few years she was examined by several physicians, who said she had heart disease. There has been nothing abnormal about menstruation since that function was established at the age of fourteen. Since that time she has never but once consulted a physician for her heart. She was married ten years ago, and has had four children. She has always suffered from cold hands and feet, and occasionally has had slight swelling of the ankles. She does her own house-work, with some help about washing and ironing.

She is a fairly strong-looking woman, of medium height, face slightly flushed, but not cyanotic or livid. Finger- and toe nails somewhat rounded, and blue (instead of the normal color) under them. On examination of the chest the most striking point is a long inspiration, like a sigh, occurring from three to six times a minute. She says she always breathes in this way, feeling as if she should smother otherwise. The night-nurse reports that there is no irregularity in her breathing when asleep. The apex beat of the heart is faint and diffused, and can be only definitely localized when she is sitting up. It is then found in the mammary line below the seventh rib. No thrill is perceptible over the heart, nor any impulse to be felt below the ensiform cartilage. The heart's dulness begins at the upper border of the fourth rib, thence to the apex beat, and thence to the middle of the sternum. Over most of the cardiac area the heart-sounds are normal, though rather faint, and there is no murmur over the aortic valves. 'To the left of the sternum, however, in the first, second and third intercostal spaces, is a soft, blowing, systolic murmur. Its point of greatest intensity is in the second intercostal space, close to the sternum, whence it may be traced upwards, and to the left, in a line pointing directly to the middle of the clavicle. Downwards it cannot be heard further than the third intercostal space. The murmur does not disappear on full inspiration. The pulmonic second sound is fainter than normal, and there is no thrill or impulse to be felt over this artery. Percussion and auscultation reveal nothing abnormal in the lungs. Dulness is normal over the liver and spleen. There is no ascites, and no œdema of any part of the body. Superficial veins of legs and upper part of the chest are well marked. There is no pulsation in the jugular veins, nor are they particularly prominent. 'The radial pulse is small and weak, has ranged between 70 and 90 , and is alike on both sides. Examination of the urine shows it to be acid, specific gravity 1,015, and containing neither albumen nor sugar. Since being in the hospital she has once spit up a little blood, or bloody mucus, which I did not see, and have no reason to suppose came from the lungs; but the fact is interesting in view of the frequent termination of such cases in phthisis, with hæmoptysis often an early symptom. Her temperature became normal the next day after her entrance, and she has shown no symptoms of typhoid fever. 